\title{
Dispatcher-assisted cardiopulmonary resuscitation for traumatic patients with out-of-hospital cardiac arrest
}

\author{
Chien-Hsin Lư ${ }^{\dagger}$ Pin-Hui Fang and Chih-Hao Lin ${ }^{*}$ (D)
}

\begin{abstract}
Background: Resuscitation efforts for traumatic patients with out-of-hospital cardiac arrest (OHCA) are not always futile. Dispatcher-assisted cardiopulmonary resuscitation (DA-CPR) during emergency calls could increase the rate of bystander cardiopulmonary resuscitation (CPR) and thus may enhance survival and neurologic outcomes of nontraumatic OHCA. This study aimed to examine the effectiveness of DA-CPR for traumatic OHCA.

Methods: A retrospective cohort study was conducted using an Utstein-style population database with data from January 1, 2014, to December 31, 2016, in Tainan City, Taiwan. Voice recordings of emergency calls were retrospectively retrieved and reviewed. The primary outcome was an achievement of sustained $(\geq 2 \mathrm{~h})$ return of spontaneous circulation (ROSC); the secondary outcomes were prehospital ROSC, ever ROSC, survival at discharge and favourable neurologic status at discharge. Statistical significance was set at a $p$-value of less than 0.05 .
\end{abstract}

Results: A total of 4526 OHCA cases were enrolled. Traumatic OHCA cases $(n=560,12.4 \%)$, compared to medical OHCA cases $(n=3966,87.6 \%)$, were less likely to have bystander CPR $(10.7 \%$ vs. $31.7 \%, p<0.001)$ and initially shockable rhythms $(7.1 \%$ vs. $12.5 \%, p<0.001)$. Regarding DA-CPR performance, traumatic OHCA cases were less likely to have dispatcher recognition of cardiac arrest ( $6.3 \%$ vs. $42.0 \%, p<0.001)$, dispatcher initiation of bystander CPR (5.4\% vs. $37.6 \%, p<0.001)$, or any dispatcher delivery of CPR instructions (2.7\% vs. $20.3 \%, p<0.001)$. Stepwise logistic regression analysis showed that witnessed cardiac arrests (aOR 1.70,95\% Cl 1.10-2.62; $p=0.017$ ) and transportation to level 1 centers (aOR 1.99, 95\% Cl 1.27-3.13; $p=0.003$ ) were significantly associated with achievement of sustained ROSC in traumatic OHCA cases, while DA-CPR-related variables were not (All $p>0.05$ ).

Conclusions: DA-CPR was not associated with better outcomes for traumatic OHCA in achieving a sustained ROSC. The DA-CPR program for traumatic OHCAs needs further studies to validate its effectiveness and practicability, especially in the communities where rules for the termination of resuscitation in prehospital settings do not exist.

Keywords: Dispatcher-assisted cardiopulmonary resuscitation, Trauma, Out-of-hospital cardiac arrest

\section{Introduction}

Out-of-hospital cardiac arrest (OHCA) is a major public health concern. Dispatch centers constitute an important link in the chain of survival for patients with OHCA $[1,2]$. Dispatcher-assisted cardiopulmonary resuscitation (DACPR) during emergency calls could increase the rate of bystander cardiopulmonary resuscitation (CPR) and thus

\footnotetext{
* Correspondence: emergency.lin@gmail.com

${ }^{\dagger}$ Chien-Hsin Lu and Chih-Hao Lin are contributed equally as first authors. Department of Emergency Medicine, National Cheng Kung University Hospital, College of Medicine, National Cheng Kung University, 70403, No.138, Shengli Rd., North District, Tainan, Taiwan
}

may enhance survival and neurologic outcomes of nontraumatic OHCA [3-5].

Traumatic OHCA generally has a higher incidence in the young population and is associated with significant mortality and grave neurological outcomes $[6,7]$. The resuscitation of traumatic OHCA is broadly considered ineffective; however, these efforts were not always futile [8]. The rate of ever achieving spontaneous return of circulation (ROSC) in traumatic OHCAs, which was up to nearly $30 \%$, could be comparable to that in medical OHCAs [6, 9, 10]. Approximately $10 \%$ of traumatic OHCA cases who survived to hospital admission had

(c) The Author(s). 2019 Open Access This article is distributed under the terms of the Creative Commons Attribution 4.0 International License (http://creativecommons.org/licenses/by/4.0/), which permits unrestricted use, distribution, and 
good neurological outcomes [11]. Survival of traumatic cardiac arrests gradually increased over years [12].

Traumatic OHCA cases, compared to medical OHCA cases, were less likely to receive bystander CPR or have resuscitation commenced by emergency medical technicians (EMTs) [13]. Despite temporal increases in rates of bystander CPR administration and paramedic resuscitation, traumatic OHCA survival remains poor [13]. Although bystander CPR is generally considered an important factor to enhance the outcomes of medical OHCAs $[14,15]$, the effect of bystander CPR on the outcomes of traumatic OHCAs is questionable [16-18]. The DA-CPR program aims to increase bystander CPR rates for OHCA cases; however, the effect of DA-CPR on traumatic OHCA cases has been examined rarely [19]. This study aimed to 1) describe the patient characteristics and DA-CPR performance in traumatic and medical OHCAs; 2) evaluate the effects of DA-CPR on traumatic OHCAs; and 3) explore the obstacles that impeded DA-CPR in traumatic OHCAs.

\section{Methods}

Study design and settings

A retrospective cohort study was conducted using an Utstein-style population database in Tainan city, Taiwan. Patients with OHCA who were transported by local emergency medical services (EMS) system between January 1, 2014, and December 31, 2016, were enrolled. Cardiac arrest was defined as the absence of signs of circulation confirmed by EMTs on the scene. Patients with a known pregnancy; who are less than 8 years old; and who have obvious signs of irreversible death, severe hypothermia, or valid do-not-attempt-resuscitation (DNAR) orders were excluded. Patients with evidence of hanging, drowning, electric shocks, or lightning strikes were also excluded [20]. The causes of cardiac arrest were classified as traumatic or medical based on the clinical judgements of the EMS providers and physicians in charge. A traumatic cardiac arrest was defined as a cardiac arrest that was consequent upon a prior traumatic event; those not classified as traumatic were defined as medical. Bystander CPR was defined as an ongoing CPR by bystanders that were confirmed by the first EMT on the scene.

\section{EMS system in Tainan city}

Tainan City constitutes an area of $2192 \mathrm{~km}^{2}$ with a population of 1.9 million. The EMS dispatch centre in Tainan is single and centralized. The annual EMS call volume in Tainan was 94,642 in 2016, which is equivalent to 13.8 calls per 100,000 population per day. The averages of EMS response time (interval of ambulance departure from the fire station to ambulance arrival at the scene), the EMS time at the scene (interval of ambulance arrival at the scene to ambulance departure from the scene), and the EMS transport time (interval of ambulance departure from the scene to ambulance arrival at the hospital) were $6.6,8.0$, and $8.2 \mathrm{~min}$, respectively. During the study period, all EMTs performed CPR according to Taiwanese guidelines based on the American Heart Association, European Resuscitation Council, and International Liaison Committee on Resuscitation 2010 Guidelines. Patients with OHCA received CPR for two full cycles (approximately $5 \mathrm{~min}$ ) before being transported to a designated hospital. Application of an automated external defibrillator was mandatory during resuscitation for medical OHCA but was optional for traumatic OHCA [16]. Resuscitation continued during transport unless ROSC was achieved. Rules for the termination of resuscitation in prehospital settings did not exist. As such, all patients with cardiac arrest who were assessed by EMTs were sent to a hospital unless obvious signs of irreversible death were present [21].

\section{DA-CPR program}

The DA-CPR program in Tainan City was initiated on June 1, 2013. As part of the Pan-Asian Resuscitation Outcome Study Phase II (PAROS-II), Tainan adopted a comprehensive DA-CPR package, which has been described in previous studies [3, 22]. All dispatchers participated in 4-h DA-CPR training course. Dispatchers were trained to comply with a streamlined, two-step question approach to identify possible cardiac arrests. The two-step question consisted of "Is the victim conscious?" and "Is the victim breathing normally?" If the callers responded that the victim was unconscious and was not breathing normally, the presumptive diagnosis was cardiac arrest, and the dispatcher initiated a protocol that delivered instructions for chest compression-only CPR.

Voice recordings of emergency calls for patients with OHCA who were confirmed by EMTs on scene were retrospectively retrieved and reviewed. A team of seven EMTs who received two courses of 4-h evaluation training reviewed the voice recordings every week. The review protocol was consistent with the Singapore version [22]. These reviewing EMTs identified barriers that impeded successful DA-CPR instructions and classified them into one or more of 15 pre-determined barrier types, which were derived from a prior literature review and a focus group discussion in Tainan City, with the provision to enter any unexpected barriers as free text [22]. A medical director then reviewed the results every 2 weeks and randomly double-checked the EMTs' reviews to ensure quality. A feedback session was conducted monthly where the medical director and dispatchers assembled to discuss the overall performance and the specific DA-CPR cases from the preceding month.

\section{Characteristics of OHCAs}

Data were obtained from a citywide OHCA registry database using paper and computer interfaces. The OHCA 
registry system was constructed using dispatch registries, EMS run registries, EMS cardiac arrest registries, and an OHCA registry for hospital care and outcomes. Collected data included the information required for the international Utstein-style criteria, which included the patient's age, gender, witness status, past medical history, EMS response time, EMS time at the scene, EMS transport time, initial cardiac rhythms, presence of bystander CPR, extent and amount of emergency care, achievement of ROSC, hospital admission, and survival and neurologic outcome at discharge [20].

The measurements of DA-CPR consisted of dispatcher recognition of cardiac arrests, dispatcher initiation of bystander CPR, any dispatcher delivery of CPR instructions (defined as the presence of CPR instruction given by dispatchers), and full dispatcher delivery of CPR instructions (defined as good quality of uninterrupted CPR instructions until the arrival of EMTs).

A comparison of traumatic and medical OHCAs was conducted [6, 13]. Categorical variables are shown as numbers and percentages, while quantitative data are shown as mean values and standard deviations (SDs). We used the chi-squared test or Fisher's exact test for categorical variables and the Mann-Whitney $\mathrm{U}$ test for continuous variables when applicable. A two-tailed $p$-value less than 0.05 was considered statistically significant.

The statistical software SPSS (version 17; SPSS Inc., Chicago, IL, USA) was used for statistical analysis.

\section{Effects of DA-CPR on traumatic OHCAs}

The primary outcome evaluated was an achievement of sustained ( $\geq 2 \mathrm{~h})$ ROSC [16]. The secondary outcomes were prehospital ROSC, ever ROSC, survival at $24 \mathrm{~h}$, survival at discharge, and good neurological status at discharge defined by the cerebral performance category (CPC) scale I or II [16].

The odds ratio (OR) and its 95\% confidence interval (CI) were used as the outcome measures. Univariate analysis was conducted to examine the association of variables and the primary outcome. Multivariate analyses were performed to examine the association and interaction among independent variables and the primary outcome. A stepwise variable selection procedure was applied to obtain the final logistic regression model. Significance levels for entry and to remain were set at 0.15 to avoid the exclusion of potential candidate variables. The final logistic regression model was identified by sequentially excluding individual variables with a $p$-value $>0.05$ until all regression coefficients were significant. If the variable of interest, i.e., DA-CPR-related variables, was excluded during the model-fitting process, it was forcibly entered in the final regression model to estimate the effect on the outcomes.

\section{Obstacles that impeded DA-CPR}

The obstacles that impeded DA-CPR for traumatic and medical OHCAs were reviewed and compared [23, 24].

\section{Ethical consideration}

The study was in accordance with ethical standards and was approved by the Institutional Review Board in National Cheng Kung University Hospital (B-ER-107-228).

\section{Results}

\section{Study objects}

Among the 7304 EMS-assessed OHCA cases during the study period, 4526 cases were included in the analysis after excluding paediatric (<8 years) patients $(n=37)$, obvious deaths $(n=2048)$, hangings $(n=65)$, drownings $(n=61)$, lightning strikes $(n=6)$, and missing data $(n=$ 561). Of the OHCA cases enrolled, 560 (12.4\%) were considered traumatic and $3966(87.6 \%)$ were considered medical. Figure 1 provides an overview of OHCA cases evaluated during the study period.

\section{Characteristics of OHCAs}

The demographic findings of OHCA cases are described in Table 1. Compared to medical OHCA cases, traumatic OHCA cases were significantly younger $(52.5 \pm$

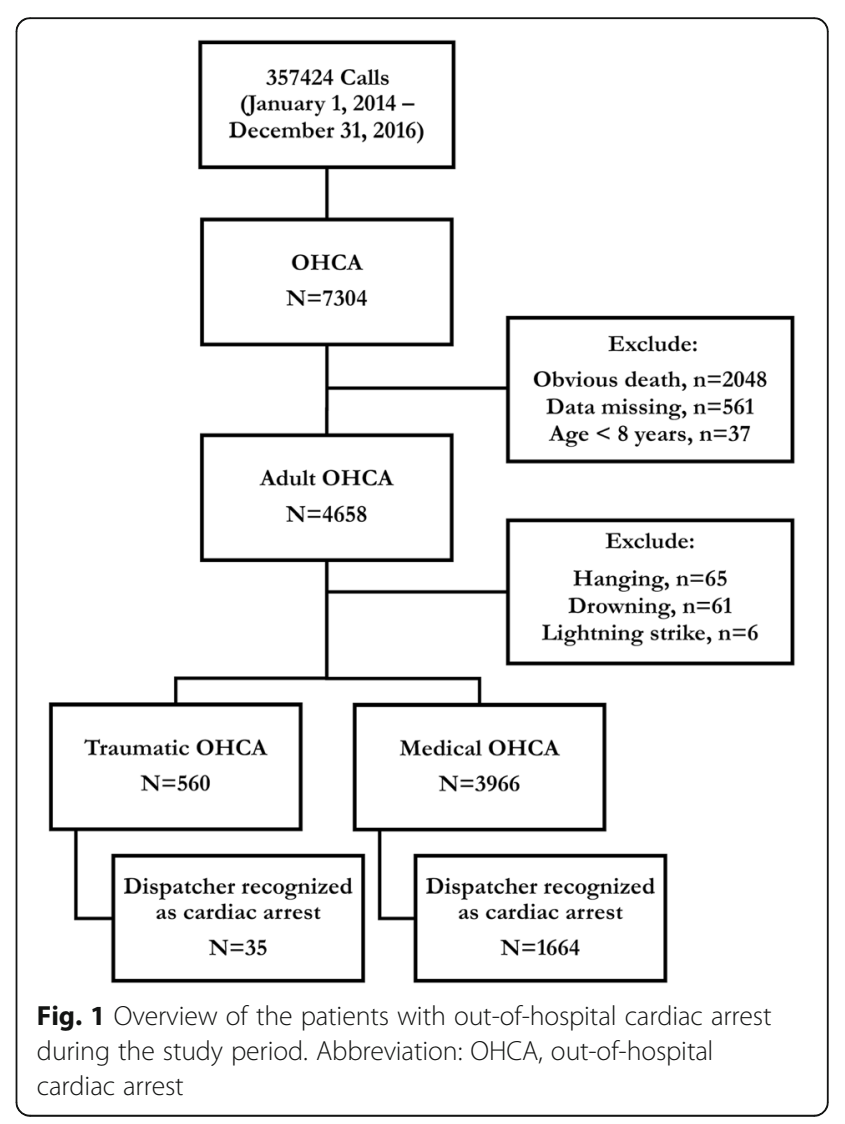


Table 1 Characteristics of patients with out-of-hospital cardiac arrest

\begin{tabular}{|c|c|c|c|}
\hline & Traumatic OHCA $(N=560)$ & Medical OHCA $(N=3966)$ & $P$ value \\
\hline Gender (Male) & $413(73.5 \%)$ & $2530(63.3 \%)$ & $<0.001$ \\
\hline \multicolumn{4}{|l|}{ Age } \\
\hline Years, mean $( \pm S D)$ & $52.5( \pm 19.7)$ & $69.2( \pm 16.6)$ & $<0.001$ \\
\hline$>55$ years $(n, \%)$ & $255(45.5 \%)$ & $3223(81.3 \%)$ & $<0.001$ \\
\hline \multicolumn{4}{|l|}{ Medical history } \\
\hline Diabetes & $40(7.1 \%)$ & 1078 (27.2\%) & $<0.001$ \\
\hline Hypertension & $57(10.2 \%)$ & 1438 (36.3\%) & $<0.001$ \\
\hline Malignancy & $10(1.8 \%)$ & $469(11.8 \%)$ & $<0.001$ \\
\hline COPD/Asthma & $16(2.9 \%)$ & $300(7.6 \%)$ & $<0.001$ \\
\hline Stroke & $9(1.6 \%)$ & $418(10.5 \%)$ & $<0.001$ \\
\hline Liver disease & $8(1.4 \%)$ & $141(3.6 \%)$ & 0.008 \\
\hline Renal disease & $14(2.5 \%)$ & $449(11.3 \%)$ & $<0.001$ \\
\hline Heart disease & $33(5.9 \%)$ & $902(22.7 \%)$ & $<0.001$ \\
\hline Witnessed cardiac arrest & $272(48.6 \%)$ & 1948 (49.1\%) & 0.809 \\
\hline By public & $148(54.4 \%)$ & $184(9.4 \%)$ & $<0.001$ \\
\hline By families & $51(18.8 \%)$ & $1336(68.6 \%)$ & $<0.001$ \\
\hline Bystander CPR & $60(10.7 \%)$ & 1257 (31.7\%) & $<0.001$ \\
\hline Breathing and chest compression & $9(15.0 \%)$ & $209(16.6 \%)$ & 0.740 \\
\hline Breathing only & $3(5.0 \%)$ & $16(1.3 \%)$ & 0.052 \\
\hline Chest compression only & $48(80.0 \%)$ & $1032(82.1 \%)$ & 0.679 \\
\hline EMS response time (mean $\pm S D$, sec) & $434.6(243.7)$ & $411.2(202.6)$ & 0.013 \\
\hline EMS scene time (mean $\pm S D$, sec) & $658.6(499.7)$ & $651.4(370.5)$ & 0.682 \\
\hline EMS transport time (mean $\pm S D$, sec) & $457.8(483.0)$ & $385.8(420.7)$ & $<0.001$ \\
\hline Prehospital use of laryngeal mask airway & $398(71.1 \%)$ & $3175(80.1 \%)$ & $<0.001$ \\
\hline Prehospital use of epinephrine & $18(3.2 \%)$ & $173(4.4 \%)$ & 0.206 \\
\hline Use of automated external defibrillator & $449(80.2 \%)$ & 3650 (92.0\%) & $<0.001$ \\
\hline Shockable rhythms & $32(7.1 \%)$ & $455(12.5 \%)$ & $<0.001$ \\
\hline Sent to Level 1 centers & $148(26.4 \%)$ & $1043(26.3 \%)$ & 0.964 \\
\hline \multicolumn{4}{|l|}{ Outcomes } \\
\hline Prehospital ROSC & $20(3.6 \%)$ & $182(4.6 \%)$ & 0.274 \\
\hline Ever ROSC & $131(23.4 \%)$ & $897(22.6 \%)$ & 0.682 \\
\hline Sustained ( $\geq 2 \mathrm{~h}$ ) ROSC & $105(18.8 \%)$ & $837(21.1 \%)$ & 0.199 \\
\hline Survival at $24 \mathrm{~h}$ & $75(13.4 \%)$ & $660(16.6 \%)$ & 0.051 \\
\hline Survival at discharge & $11(2.0 \%)$ & $181(4.6 \%)$ & 0.004 \\
\hline Good neurologic outcome at discharge & $4(0.7 \%)$ & $110(2.8 \%)$ & 0.002 \\
\hline
\end{tabular}

Abbreviations: OHCA out-of-hospital cardiac arrest; SD standard deviation; COPD chronic obstructive pulmonary disease; CPR cardiopulmonary resuscitation; EMS emergency medical services; ROSC return of spontaneous circulation

19.7 years vs. $69.2 \pm 16.6$ years, $p<0.001)$ and maledominant $(73.3 \%$ vs. $63.3 \%, p<0.001)$. Traumatic OHCA cases were more likely to be witnessed by the public (54.4\% vs. $9.4 \%, p<0.001)$ but less likely to be witnessed by families $(18.8 \%$ vs. $68.6 \%, p<0.001)$. Traumatic OHCA cases were less likely to have bystander CPR (10.7\% vs. $31.7 \%, p<0.001)$ and initially shockable rhythms (7.1\% vs. $12.5 \%, p<0.001)$.
There were no statistical significances between the traumatic and medical OHCA cases regarding the achievements of prehospital ROSC (3.6\% vs. $4.6 \%)$, ever ROSC (23.4\% vs. $22.6 \%$ ), sustained ( $\geq 2$ h) ROSC (18.8\% vs. $21.1 \%)$, or survival at $24 \mathrm{~h}(13.4 \%$ vs. $16.6 \%)$ (All $p \geq 0.05)$. Compared to medical OHCA cases, traumatic OHCA cases were less likely to survive $(2.0 \%$ vs. $4.6 \%, p=0.004)$ and show good neurologic outcomes $(0.7 \%$ vs. $2.8 \%, p=0.002)$ at discharge. 


\section{DA-CPR performance}

Table 2 describes DA-CPR performance for OHCA cases. Dispatchers were less likely to comply with dispatch protocols for traumatic OHCA cases $(14.6 \%$ vs. $59.4 \%, p<0.001)$. Traumatic OHCA cases were less likely to have dispatcher recognition of cardiac arrest (6.3\% vs. $42.0 \%)$, dispatcher initiation of bystander CPR (5.4\% vs. $37.6 \%)$, or any dispatcher delivery of CPR instructions $(2.7 \%$ vs. $20.3 \%)$ (All $p<0.001)$.

\section{Effects of DA-CPR on traumatic OHCAs}

Traumatic OHCA cases that were identified as cardiac arrests by dispatchers $(n=35)$, compared to medical OHCA cases $(n=525)$, had higher rates of bystander CPR $(37.1 \%$ vs. $9.0 \%, p<0.001)$ and more initially shockable rhythms $(14.3 \%$ vs. $5.1 \%, p=0.024)$. There were no significant differences between the two groups regarding any outcome measure (Table 3).

Table 4 shows the effects of key variables on the achievement of sustained $(\geq 2 \mathrm{~h})$ ROSC in traumatic OHCA cases. Dispatcher recognition of cardiac arrests, dispatcher initiation of bystander CPR, any dispatcher delivery of CPR instructions, full dispatcher delivery of CPR instructions, or presence of bystander CPR had statistically insignificant effects in the univariate and multivariate models (all $p>0.05$ ). The stepwise logistic regressions showed that witnessed cardiac arrests (adjusted OR [aOR] 1.70, 95\% CI 1.10-2.62; $p=0.017$ ) and transportation to level 1 centers (aOR 1.99, 95\% CI 1.27-3.13; $p=0.003$ ) were significantly associated with the achievement of sustained ROSC in traumatic OHCA cases, while DA-CPR-related variables were not (All $p>$ 0.05).

The logistic regression analysis showed that none of the DA-CPR-related variables was significantly associated with the secondary outcomes (that is, prehospital ROSC, ever ROSC, survival at $24 \mathrm{~h}$, survival at discharge, or good neurological status at discharge) (All $p>0.05)$.

\section{Obstacles that impeded DA-CPR}

Patients with OHCA that did not receive full dispatcher delivery of CPR instructions were eligible for further analysis to identify the obstacles that impeded DA-CPR (Table 5). The obstacles in traumatic OHCA cases $(n=$ 559), compared to medical OHCA cases $(n=3926)$, were more likely associated with "callers physically distant from the victims" (34.0\% vs. 10.0\%), "difficulty of access to the victims" (30.1\% vs. 19.4\%;), "third party callers such as policepersons" ( $11.1 \%$ vs. $2.3 \%)$, or "dangerous scenes" $(5.2 \%$ vs. $0.2 \%)$ (All $p<0.001)$.

\section{Discussion}

The results of our study found that the compliance with and performance of DA-CPR for traumatic OHCA cases were poor. The rates of dispatcher recognition of cardiac arrest, dispatcher initiation of bystander CPR, or any dispatcher delivery of CPR instructions were not associated with the achievement of sustained ROSC in traumatic OHCA cases.

Our findings may imply that the concept of chain of survival in traumatic OHCA could be different from that in medical OHCA. In general, medical OHCA events that occur in public locations are more likely to receive bystander CPR than are those in non-public locations [25]. Patients with medical OHCA may receive more resuscitation when they are witnessed by non-family bystanders than by family members [26]. Traumatic OHCA events, compared to medical OHCA events, were more likely to occur in public locations; however, the rate of bystander CPR for traumatic OHCA cases was extremely low. The 119 callers for traumatic OHCA events that occurred in public locations were often not physically alongside the patients $[22,27,28]$. The dispatchers could ask the callers to move beside the victim and redial emergency calls by a mobile phone [29]. However, the bystanders may call 119 after they have already left the scene and may hesitate when asked to approach the traumatic patient. Concerns of bloodborne diseases and safety issues in trauma scenes

Table 2 Dispatcher-assisted cardiopulmonary resuscitation for patients with out-of-hospital cardiac arrest

\begin{tabular}{|c|c|c|c|}
\hline & $\begin{array}{l}\text { Traumatic OHCA } \\
(N=560)\end{array}$ & $\begin{array}{l}\text { Medical OHCA } \\
(N=3966)\end{array}$ & $P$ value \\
\hline \multicolumn{4}{|l|}{ Compliance of dispatch protocol } \\
\hline Dispatchers evaluate both the consciousness and presence of normal breathing & $82(14.6 \%)$ & $2355(59.4 \%)$ & $<0.001$ \\
\hline Dispatchers only evaluate the consciousness & $107(19.1 \%)$ & $2642(66.6 \%)$ & $<0.001$ \\
\hline Dispatchers only evaluate the presence of normal breathing & $99(17.7 \%)$ & $2751(69.4 \%)$ & $<0.001$ \\
\hline Dispatcher-recognition of cardiac arrests & $35(6.3 \%)$ & $1664(42.0 \%)$ & $<0.001$ \\
\hline Dispatcher-initiation of bystander CPR & $30(5.4 \%)$ & $1491(37.6 \%)$ & $<0.001$ \\
\hline Any dispatcher-delivery of CPR instructions & $15(2.7 \%)$ & $806(20.3 \%)$ & $<0.001$ \\
\hline Full dispatcher-delivery of CPR instructions & $1(0.2 \%)$ & $40(1.0 \%)$ & 0.054 \\
\hline
\end{tabular}

Abbreviation: $C P R$ cardiopulmonary resuscitation 
Table 3 Characteristics of traumatic patients with out-of-hospital cardiac arrest who were and were not recognized as cardiac arrest by dispatchers

\begin{tabular}{|c|c|c|c|}
\hline & Dispatcher $r$ & & \\
\hline & Yes $(N=35)$ & No $(N=525)$ & $P$ value \\
\hline Gender (Male) & $24(68.6 \%)$ & $388(73.9 \%)$ & 0.488 \\
\hline Age & & & \\
\hline Years, mean $( \pm \mathrm{SD})$ & $58.6( \pm 20.1)$ & $51.9( \pm 19.6)$ & 0.051 \\
\hline$>55$ years $(n, \%)$ & $20(57.1 \%)$ & $235(44.6 \%)$ & 0.154 \\
\hline Mechanism & & & 1.000 \\
\hline Penetrating injury & 0 & $6(1.1 \%)$ & \\
\hline Blunt injury & $35(100 \%)$ & $519(98.9 \%)$ & \\
\hline Witnessed cardiac arrest & $15(42.9 \%)$ & $257(49.0 \%)$ & 0.485 \\
\hline Bystander CPR & $13(37.1 \%)$ & $47(9.0 \%)$ & $<0.001$ \\
\hline Prehospital use of laryngeal mask airway & $24(68.6 \%)$ & $374(71.2 \%)$ & 0.736 \\
\hline Prehospital use of epinephrine & 0 & $18(3.4 \%)$ & 0.619 \\
\hline Use of automated external defibrillator & $30(85.7 \%)$ & 419 (79.8\%) & 0.396 \\
\hline Initially shockable rhythms & $5(14.3 \%)$ & $27(5.1 \%)$ & 0.042 \\
\hline Sent to level 1 centers & $137(26.1 \%)$ & $148(26.4 \%)$ & 0.488 \\
\hline Outcomes & & & \\
\hline Prehospital ROSC & $1(2.9 \%)$ & $19(3.6 \%)$ & 1.000 \\
\hline Ever ROSC & $6(17.1 \%)$ & $125(23.8 \%)$ & 0.367 \\
\hline Sustained $(\geq 2)$ ROSC & $6(17.1 \%)$ & $101(19.2 \%)$ & 0.760 \\
\hline Survival at $24 \mathrm{~h}$ & $4(11.4 \%)$ & $72(13.7 \%)$ & 0.702 \\
\hline Survival at discharge & $1(2.9 \%)$ & $10(1.9 \%)$ & 0.694 \\
\hline Good neurologic outcomes at discharge & 1 (2.9\%) & $3(0.6 \%)$ & 0.120 \\
\hline
\end{tabular}

Abbreviations: OHCA out-of-hospital cardiac arrest; SD standard deviation; CPR cardiopulmonary resuscitation; ROSC return of spontaneous circulation

Table 4 Unadjusted (univariate model) and adjusted (logistic regression model) odds ratios for achieving a sustained ( $\geq 2 \mathrm{~h}$ ) return of spontaneous circulation in traumatic patients with out-of-hospital cardiac arrest

\begin{tabular}{|c|c|c|c|c|}
\hline & \multicolumn{2}{|c|}{ Unadjusted analysis } & \multicolumn{2}{|l|}{ Adjusted analysis } \\
\hline & OR (95\% Cls) & $P$ value & $\mathrm{aOR}(95 \% \mathrm{Cls})$ & $P$ value \\
\hline Male & $0.99(0.61-1.59)$ & 0.951 & & \\
\hline Age (8-55 years) & $1.15(0.75-1.78)$ & 0.528 & & \\
\hline Penetrating trauma & $2.19(0.40-12.12)$ & 0.357 & & \\
\hline Witnessed cardiac arrest & $1.68(1.09-2.59)$ & 0.017 & $1.70(1.10-2.62)$ & 0.017 \\
\hline Dispatcher-recognition of cardiac arrests & $0.89(0.36-2.20)$ & 0.801 & $1.02(0.18-5.99)$ & 0.979 \\
\hline Dispatcher-initiation of bystander CPR & $0.86(0.32-2.30)$ & 0.764 & $0.61(0.07-5.13)$ & 0.650 \\
\hline Any dispatcher-delivery of CPR instructions & $1.09(0.30-3.92)$ & 0.900 & $0.80(0.06-10.81)$ & 0.868 \\
\hline Full dispatcher-delivery of CPR instructions & $2.19(0.40-12.12)$ & 0.357 & $4.51(0.29-69.41)$ & 0.280 \\
\hline Bystander CPR & $1.09(0.56-2.14)$ & 0.793 & & \\
\hline Prehospital use of epinephrine & $1.25(0.40-3.87)$ & 0.701 & & \\
\hline Initially shockable rhythms & $1.76(0.79-3.93)$ & 0.162 & & \\
\hline Prehospital use of laryngeal mask airway & $0.91(0.58-1.45)$ & 0.698 & & \\
\hline Sent to level 1 centers & $1.98(1.26-3.10)$ & 0.003 & $1.99(1.27-3.13)$ & 0.003 \\
\hline
\end{tabular}


Table 5 Analysis of obstacles that impeded dispatcher-assisted cardiopulmonary resuscitation for patients with out-of-hospital cardiac arrest

\begin{tabular}{|c|c|c|c|}
\hline & Traumatic OHCA $(N=559)$ & Medical OHCA $(N=3926)$ & $P$ value \\
\hline Caller not beside the victim & $340(60.8 \%)$ & $1082(27.6 \%)$ & $<0.001$ \\
\hline Caller not physically alongside the victim & $190(34.0 \%)$ & $393(10.0 \%)$ & $<0.001$ \\
\hline Difficult access to the victim & $168(30.1 \%)$ & $760(19.4 \%)$ & $<0.001$ \\
\hline Third party caller & $62(11.1 \%)$ & $90(2.3 \%)$ & $<0.001$ \\
\hline Dangerous scene & $29(5.2 \%)$ & $9(0.2 \%)$ & $<0.001$ \\
\hline Dispatcher can't recognize the need for CPR & $253(45.3 \%)$ & $1306(33.3 \%)$ & $<0.001$ \\
\hline Caller hanged up & $16(2.9 \%)$ & $124(3.2 \%)$ & 0.706 \\
\hline Overly distraught & $15(2.7 \%)$ & $124(3.2 \%)$ & 0.544 \\
\hline Dispatcher hanged up & $12(2.1 \%)$ & $74(1.9 \%)$ & 0.673 \\
\hline Patient's status changed & $7(1.3 \%)$ & $97(2.5 \%)$ & 0.073 \\
\hline Caller is unable to perform CPR & $4(0.7 \%)$ & $12(0.3 \%)$ & 0.129 \\
\hline Caller is unable to move the patient & $4(0.7 \%)$ & $2(0.1 \%)$ & 0.003 \\
\hline CPR is already ongoing & $2(0.4 \%)$ & $14(0.4 \%)$ & 1.000 \\
\hline Caller refused to perform CPR & $1(0.2 \%)$ & $6(0.2 \%)$ & 0.606 \\
\hline Valid consent of Do-Not-Attempt-Resuscitate orders & 0 & 0 & - \\
\hline
\end{tabular}

Abbreviations: OHCA out-of-hospital cardiac arrest, CPR cardiopulmonary resuscitation

could impede the bystanders from providing a physical assessment and even resuscitation efforts [30].

The bystander CPR rate could significantly increase if dispatchers could recognize the need to perform CPR on the patient, even in traumatic OHCA cases. Compared with those not recognized by dispatchers, traumatic OHCA cases that were recognized by dispatchers had more bystander $\mathrm{CPR}$ and more initially shockable rhythms. We were unable to evaluate the characteristics of the bystanders. Their willingness to stay alongside the traumatic patients with OHCA and to perform resuscitation at the scene is an important issue to investigate. We suspect that the degree of chaos at the trauma scene and the apparent severity of injury could influence the likelihood of bystander CPR.

The compliance of DA-CPR protocols was generally poor when the dispatchers evaluated the calls regarding traumatic patients. Severe traumatic incidents may give dispatchers the impression that the prognosis of victims with traumatic OHCAs will be grave even when resuscitation efforts are performed, which may consequently result in poor compliance with DA-CPR protocols and thus lower the recognition rate of cardiac arrest.

The reason that dispatchers were unable to correctly identify cardia arrest in traumatic cases deserves further exploration. In this study, the dispatchers used the same protocol to identify possible cardiac arrests in either traumatic or medical patients. The protocol consisted of a streamlined, two-step question (that is, "Is the victim conscious?" and "Is the victim breathing normally?") Although the two-step question performs well in identifying medical
OHCA $[3,22]$, its practicability and effectiveness to identify cardiac arrest in traumatic patients are rarely examined [19]. We suspected that the optimal protocol for dispatchers to identify cardiac arrest in traumatic patients could be different from that in medical patients.

Once the dispatchers recognized an event of OHCA in emergency calls, the rate of initiating and executing CPR instructions for traumatic OHCA patients and medical OHCA patients were similar. We did not evaluate the personality characteristics or telephone skills of individual dispatchers. We assumed that the dispatchers who recognized cardiac arrests in traumatic patients were more aggressive in providing resuscitation efforts, which may have thus resulted in a type 1 error with better outcomes for traumatic OHCA patients. However, the study results did not find improved outcomes in the group.

The policy of "resuscitation during transportation" is not common in Western EMS systems. However, due to the lack of rules for termination of resuscitation in the prehospital settings, many Asian EMS systems utilize the protocols of resuscitation during transportation [21]. A recent study found that high quality CPR metrics (including chest compression fraction, compression rate, and compression depth) were similar at the scene and during ambulance transportation [31]. High quality CPR can be performed by prehospital providers regardless of location [31]. The evolution of mechanical CPR and other devices could have impacts on current EMS protocols of managing OHCAs [32].

Our study results showed that bystander CPR, initially shockable rhythms and advanced life supports, such as 
the use of epinephrine or an advanced airway, were not significantly associated with the achievement of sustained ROSC. We only found that witnessed cardiac arrests and transportation to level 1 centers were significantly associated with the primary outcome. These findings imply that the traditional "load and go" model for traumatic patients might strategically apply to patients with traumatic OHCA as well $[13,16,33]$. The concept of "chain of survival" for traumatic OHCA patients deserves further exploration.

This study had several limitations. First, due to the low crime rate and strict weapon control policy, patients with traumatic OHCA who had penetrative injuries, such as stabbings or gunshots, were extremely rare in the city during the study period. Thus, our study findings might mainly apply in patients with blunt trauma. Second, this observational study was conducted in a setting with suboptimal compliance of DA-CPR protocols for traumatic OHCA cases. Although factors were adjusted using multivariable analysis, other confounding factors might exist given that this was not a randomized controlled trial. Third, we were unable to validate the actual performance and measure the quality of bystander CPR through the voice recordings in dispatch centers. This technical difficulty may require a more comprehensive system using emergency calls with real-time video communication in the future. Fourth, we were unable to approach the treatment and management of patients in receiving hospitals. The management in individual hospitals might be associated with patient outcomes. Potential limitations could also exist in multi-site studies regarding data integrity, validity, and ascertainment bias. In attempts to minimize these potential sources of bias, this populationbased cohort study utilized the time synchronization process, consistent definitions, uniform data collection, and a large sample size. Finally, this study was conducted in an EMS system that utilizes the policy of "resuscitation during transportation" for OHCAs. Thus, the application of our study results should be tailored to local EMS practices.

\section{Conclusion}

Our study results found that DA-CPR was not associated with better outcomes for traumatic OHCAs in achieving a short-term sustained ROSC. The DA-CPR program for traumatic OHCAs needs further studies to validate its effectiveness and practicability, especially in the communities where rules for the termination of resuscitation in prehospital settings do not exist.

\footnotetext{
Abbreviations

Cl: Confidence interval; CPC: Cerebral performance category; CPR: Cardiopulmonary resuscitation; DA-CPR: Dispatcher-assisted cardiopulmonary resuscitation; DNAR: Do-not-attempt-resuscitation; EMS: Emergency medical service; EMT: Emergency medical technician; OHCA: Out-of-hospital cardiac arrest; OR: Odds ratio; PAROS-II: Pan-Asian Resuscitation Outcome Study Phase II; ROSC: Return to spontaneous circulation; SD: Standard deviation
}

Acknowledgements

We would like to thank Tainan City Fire Bureau for their dedication.

\section{Authors' contributions}

$\mathrm{C}-\mathrm{HL}$ in conceived the study and developed the study protocols. C-HLu, PHF and C-HLin provided technical and administrative supports and contributed to acquisition of data. C-HLu performed statistical analysis. C-HLu and C-HLin interpreted the study results. C-HLu and C-HLin drafted the manuscript. All authors contributed substantially to its revision and approved the final manuscript. C-HLu and C-HLin contributed equally as first authors. C-HLin is the corresponding author who takes responsibility for the paper as a whole.

\section{Funding}

This study was supported by the Taiwan Ministry of Science and Technology (MOST 105-2314-B-006 -069, MOST 106-2314-B-006-003 and MOST 107-

2314-B-006-003).

\section{Availability of data and materials}

The datasets used and/or analyzed during the current study are available from the corresponding author on reasonable request.

\section{Ethics approval and consent to participate}

The study protocol was reviewed and approved by the Institutional Review Board of National Cheng Kung University Hospital, Tainan, Taiwan (B-ER-107-228).

\section{Consent for publication}

Not applicable.

\section{Competing interests}

The authors report no conflicts of interest. The authors alone are responsible for the content and writing of the paper.

Received: 16 August 2019 Accepted: 17 October 2019

Published online: 01 November 2019

\section{References}

1. Ng YY, Leong SH, Ong ME. The role of dispatch in resuscitation. Singap Med J. 2017;58:449-52.

2. Ageron FX, Debaty G, Gayet-Ageron A, Belle L, Gaillard A, Monnet MF, et al. Impact of an emergency medical dispatch system on survival from out-ofhospital cardiac arrest: a population-based study. Scand J Trauma Resusc Emerg Med. 2016;24:53.

3. Harjanto S, Na MX, Hao Y, Ng YY, Doctor N, Goh ES, et al. A before-after interventional trial of dispatcher-assisted cardio-pulmonary resuscitation for out-of-hospital cardiac arrests in Singapore. Resuscitation. 2016;102:85-93.

4. Ro YS, Shin SD, Lee YJ, Lee SC, Song KJ, Ryoo HW, et al. Effect of dispatcherassisted cardiopulmonary resuscitation program and location of out-ofhospital cardiac arrest on survival and neurologic outcome. Ann Emerg Med. 2017:69:52-61 e1.

5. Viereck S, Moller TP, Rothman JP, Folke F, Lippert FK. Recognition of out-ofhospital cardiac arrest during emergency calls - a systematic review of observational studies. Scand J Trauma Resusc Emerg Med. 2017;25:9.

6. Deasy C, Bray J, Smith K, Harriss L, Morrison C, Bernard S, et al. Traumatic out-of-hospital cardiac arrests in Melbourne. Aust Resusc. 2012;83:465-70.

7. Stockinger ZT, McSwain NE Jr. Additional evidence in support of withholding or terminating cardiopulmonary resuscitation for trauma patients in the field. J Am Coll Surg. 2004;198:227-31.

8. Barnard E, Yates D, Edwards A, Fragoso-Iñiguez M, Jenks T, Smith JE. Epidemiology and aetiology of traumatic cardiac arrest in England and Wales - a retrospective database analysis. Resuscitation. 2017;110:90-4.

9. Beck B, Bray JE, Cameron P, Straney L, Andrew E, Bernard S, et al. Predicting outcomes in traumatic out-of-hospital cardiac arrest: the relevance of Utstein factors. Emerg Med J. 2017;34:786-92.

10. Grasner JT, Wnent J, Seewald S, Meybohm P, Fischer M, Paffrath T, et al. Cardiopulmonary resuscitation traumatic cardiac arrest--there are survivors. An analysis of two national emergency registries. Crit Care. 2011;15:R276.

11. Duchateau FX, Hamada S, Raux M, Gay M, Mantz J, Paugam Burtz C, et al. Long-term prognosis after out-of-hospital resuscitation of cardiac arrest in trauma patients: prehospital trauma-associated cardiac arrest. Emerg Med J. 2017:34:34-8. 
12. Djarv T, Axelsson C, Herlitz J, Stromsoe A, Israelsson J, Claesson A. Traumatic cardiac arrest in Sweden 1990-2016 - a population-based national cohort study. Scand J Trauma Resusc Emerg Med. 2018;26:30.

13. Beck B, Tohira H, Bray JE, Straney L, Brown E, Inoue M, et al. Trends in traumatic out-of-hospital cardiac arrest in Perth, Western Australia from 1997 to 2014. Resuscitation. 2016;98:79-84.

14. Kragholm K, Wissenberg M, Mortensen RN, Hansen SM, Malta Hansen C, Thorsteinsson K, et al. Bystander efforts and 1-year outcomes in out-ofhospital cardiac arrest. N Engl J Med. 2017;376:1737-47.

15. Malta Hansen C, Kragholm K, Pearson DA, Tyson C, Monk L, Myers B, et al. Association of bystander and first-responder intervention with survival after out-of-hospital cardiac arrest in North Carolina, 2010-2013. JAMA. 2015;314:255-64

16. Lin $\mathrm{CH}$, Chiang WC, Ma MH, Wu SY, Tsai MC, Chi CH. Use of automated external defibrillators in patients with traumatic out-of-hospital cardiac arrest. Resuscitation. 2013;84:586-91.

17. Smith JE, Rickard A, Wise D. Traumatic cardiac arrest. J R Soc Med. 2015;108:11-6.

18. Beck B, Bray JE, Cameron P, Straney L, Andrew E, Bernard S, et al. Resuscitation attempts and duration in traumatic out-of-hospital cardiac arrest. Resuscitation. 2017;111:14-21.

19. Bakke HK, Steinvik T, Ruud H, Wisborg T. Effect and accuracy of emergency dispatch telephone guidance to bystanders in trauma: post-hoc analysis of a prospective observational study. Scand J Trauma Resusc Emerg Med. 2017;25:27.

20. Perkins GD, Jacobs IG, Nadkarni VM, Berg RA, Bhanji F, Biarent D, et al. Cardiac arrest and cardiopulmonary resuscitation outcome reports: update of the Utstein Resuscitation Registry Templates for Out-of-Hospital Cardiac Arrest: a statement for healthcare professionals from a task force of the International Liaison Committee on Resuscitation (American Heart Association, European Resuscitation Council, Australian and New Zealand Council on Resuscitation, Heart and Stroke Foundation of Canada, InterAmerican Heart Foundation, Resuscitation Council of Southern Africa, Resuscitation Council of Asia); and the American Heart Association Emergency Cardiovascular Care Committee and the Council on Cardiopulmonary, Critical Care, Perioperative and Resuscitation. Circulation. 2015;132:1286-300.

21. Lin $\mathrm{CH}, \mathrm{Ng} Y \mathrm{Y}$, Chiang WC, Karim SA, Shin SD, Tanaka H, et al. Variation of current protocols for managing out-of-hospital cardiac arrest in prehospital settings among Asian countries. J Formos Med Assoc. 2016;115:628-38.

22. Ho AF, Sim ZJ, Shahidah N, Hao Y, Ng YY, Leong BS, et al. Barriers to dispatcher-assisted cardiopulmonary resuscitation in Singapore. Resuscitation. 2016;105:149-55.

23. Lindstrom V, Heikkila K, Bohm K, Castren M, Falk AC. Barriers and opportunities in assessing calls to emergency medical communication centre--a qualitative study. Scand J Trauma Resusc Emerg Med. 2014;22:61.

24. Alfsen D, Moller TP, Egerod I, Lippert FK. Barriers to recognition of out-ofhospital cardiac arrest during emergency medical calls: a qualitative inductive thematic analysis. Scand J Trauma Resusc Emerg Med. 2015;23:70.

25. Swor R, Khan I, Domeier R, Honeycutt L, Chu K, Compton S. CPR training and CPR performance: do CPR-trained bystanders perform CPR? Acad Emerg Med. 2006;13:596-601.

26. Fujie K, Nakata Y, Yasuda S, Mizutani T, Hashimoto K. Do dispatcher instructions facilitate bystander-initiated cardiopulmonary resuscitation and improve outcomes in patients with out-of-hospital cardiac arrest? A comparison of family and non-family bystanders. Resuscitation. 2014:85:315-9.

27. Fukushima H, Panczyk M, Spaite DW, Chikani V, Dameff C, Hu C, et al. Barriers to telephone cardiopulmonary resuscitation in public and residential locations. Resuscitation. 2016;109:116-20.

28. Idris AH, Roppolo L. Barriers to dispatcher-assisted telephone cardiopulmonary resuscitation. Ann Emerg Med. 2003;42:738-40.

29. Maeda T, Yamashita A, Myojo Y, Wato Y, Inaba H. Augmented survival of out-of-hospital cardiac arrest victims with the use of mobile phones for emergency communication under the DA-CPR protocol getting information from callers beside the victim. Resuscitation. 2016;107:80-7.

30. Dobbie F, Mackintosh AM, Clegg G, Stirzaker R, Bauld L. Attitudes towards bystander cardiopulmonary resuscitation: results from a cross-sectional general population survey. PLoS One. 2018;13:e0193391.

31. Cheskes S, Byers A, Zhan C, Verbeek PR, Ko D, Drennan IR, et al. CPR quality during out-of-hospital cardiac arrest transport. Resuscitation. 2017;114:34-9.
32. Lyon RM, Crawford A, Crookston C, Short S, Clegg GR. The combined use of mechanical CPR and a carry sheet to maintain quality resuscitation in outof-hospital cardiac arrest patients during extrication and transport. Resuscitation. 2015:93:102-6.

33. Evans CC, Petersen A, Meier EN, Buick JE, Schreiber M, Kannas D, et al. Prehospital traumatic cardiac arrest: management and outcomes from the resuscitation outcomes consortium epistry-trauma and PROPHET registries. J Trauma Acute Care Surg. 2016;81:285-93.

\section{Publisher's Note}

Springer Nature remains neutral with regard to jurisdictional claims in published maps and institutional affiliations.
Ready to submit your research? Choose BMC and benefit from:

- fast, convenient online submission

- thorough peer review by experienced researchers in your field

- rapid publication on acceptance

- support for research data, including large and complex data types

- gold Open Access which fosters wider collaboration and increased citations

- maximum visibility for your research: over $100 \mathrm{M}$ website views per year

At BMC, research is always in progress.

Learn more biomedcentral.com/submissions 